Jurnal Matematika UNAND

Vol. VIII No. 2 Hal. 101 - 107

Edisi Agustus 2019

ISSN : 2303-291X

(C)Jurusan Matematika FMIPA UNAND

\title{
SOLUSI SISTEM PERSAMAAN DIFERENSIAL LINIER ORDE FRACTIONAL DENGAN TURUNAN TIPE CAPUTO
}

\author{
NADIFA RAHADATUL 'AISY, RADHIATUL HUSNA \\ Program Studi S1 Matematika, \\ Fakultas Matematika dan Ilmu Pengetahuan Alam, Universitas Andalas, \\ Kampus UNAND Limau Manis Padang, Indonesia. \\ email : Nadifarahadatulaisy@yahoo.co.id
}

Diterima 22 Juni 2019 Direvisi 6 Juli 2019 Dipublikasikan 4 Agustus 2019

\begin{abstract}
Abstrak. Dalam makalah ini diselesaikan sistem persamaan diferensial linier orde fractional dengan turunan tipe Caputo. Teorema utama yang menyajikan bentuk umum solusi didiskusikan. Beberapa contoh yang mengilustrasikan teorema utama dipaparkan.

Kata Kunci: Sistem Persamaan Diferensial Fractional, Turunan Tipe Caputo
\end{abstract}

\section{Pendahuluan}

Diberikan suatu sistem persamaan diferensial fractional linier sebagai berikut.

$$
\frac{d^{\alpha} \mathbf{x}(t)}{d t^{\alpha}}=A \mathbf{x}(t)+B \mathbf{u}(t), \mathbf{x}(0)=\mathbf{x}_{0}
$$

dengan:

$$
\frac{d^{\alpha} f(t)}{d t^{\alpha}}=\frac{1}{\Gamma(m-\alpha)} \int_{0}^{t}(t-\tau)^{m-\alpha-1} \frac{d^{m}}{d t^{m}} f(\tau) d \tau
$$

dimana $A \in \mathbb{R}^{n \times n}, B \in \mathbb{R}^{n \times m}, \mathbf{u}(t) \in \mathbb{R}^{m}$, untuk $m-1<\alpha<m, m \in \mathbb{N}$, dan $\Gamma$ adalah fungsi gamma.

Dalam makalah ini akan diselesaikan sistem persamaan diferensial (1.1), dimana $\frac{d^{\alpha} \mathbf{x}(t)}{d t^{\alpha}}$ adalah turunan fractional tipe Caputo.

\section{Landasan Teori}

\subsection{Transformasi Laplace}

Definisi 2.1. [6] Misalkan $f(t)$ adalah suatu fungsi yang didefinisikan untuk $t>0$ dan $s \in \mathbb{R}$. Maka Transformasi Laplace dari $f(t)$, didefinisikan sebagai berikut

$$
\mathcal{L}[f(t)]=F(s)=\int_{0}^{\infty} f(t) e^{-s t} d t .
$$


Transformasi Laplace dari $F(s)$ dikatakan ada apabila integral pada Definisi 2.1 konvergen untuk beberapa nilai s, bila tidak demikian maka transformasi Laplace dari $F(s)$ dikatakan tidak ada [6].

Teorema 2.2. [4] Transformasi Laplace dari turunan orde ke-m dari suatu fungsi $f(t)$ diberikan sebagai berikut

$$
\mathcal{L}\left[f^{(m)}(t)\right]=s^{m} F(s)-s^{(m-1)} f(0)-s^{(m-2)} f^{\prime}(0)-\cdots-f^{(m-1)}(0) .
$$

Teorema 2.3. [3] Jika transformasi Laplace dari fungsi $f(t)$ dan $g(t)$ adalah $F(s)$ dan $G(s)$ maka transformasi Laplace untuk konvolusi kedua fungsi tersebut adalah

$$
\mathcal{L}\left[\int_{0}^{t} f(t-\tau) g(\tau) d \tau\right]=F(s) * G(s) .
$$

\subsection{Fungsi Gamma dan Fungsi Beta}

Definisi 2.4. [4] Fungsi Gamma didefinisikan sebagai berikut.

$$
\Gamma(n)=\int_{0}^{\infty} t^{n-1} e^{-t} d t, n>0 .
$$

Definisi 2.5. [4] Fungsi Beta didefinisikan sebagai berikut.

$$
B(p, q)=\int_{0}^{1} x^{p-1}(1-x)^{q-1} d x, x \in \mathbb{R}, p, q \in \mathbb{C},
$$

dimana $p>0$ dan $q>0$.

\subsection{Turunan Fractional Tipe Caputo}

Definisi 2.6. [3] Turunan fractional Caputo orde $\alpha \in \mathbb{R}$ dari fungsi $f(t)$ dengan $m-1<\alpha<m, m \in \mathbb{N}$, dinotasikan dengan $\frac{d^{\alpha} f(t)}{d t^{\alpha}}$, didefinisikan sebagai berikut.

$$
\frac{d^{\alpha} f(t)}{d t^{\alpha}}=\frac{1}{\Gamma(m-\alpha)} \int_{0}^{t} \frac{f^{(m)}(\tau) d \tau}{(t-\tau)^{\alpha+1-m}},
$$

dimana $f^{(m)}(\tau)$ adalah turunan ke-m dari fungsi $f(\tau)$ dan $\Gamma(m-\alpha)$ menyatakan fungsi gamma dari $(m-\alpha)$.

Teorema 2.7. [3] Turunan fractional Caputo memenuhi sifat kelinieran, yaitu

$$
\frac{d^{\alpha}}{d t^{\alpha}}[\lambda f(t)+\mu g(t)]=\lambda \frac{d^{\alpha}}{d t^{\alpha}} f(t)+\mu \frac{d^{\alpha}}{d t^{\alpha}} g(t), \lambda, \mu \in \mathbb{R} .
$$

Teorema 2.8. [3] Transformasi Laplace dari turunan fractional Caputo adalah

$$
\mathcal{L}\left[\frac{d^{\alpha}}{d t^{\alpha}} f(t)\right]=s^{\alpha} F(s)-\sum_{k=1}^{m} s^{(\alpha-k)} f^{(k-1)}(0) .
$$


Bukti.

$$
\begin{aligned}
\mathcal{L}\left[\frac{d^{\alpha}}{d t^{\alpha}} f(t)\right] & =\mathcal{L}\left[\frac{1}{\Gamma(m-\alpha)} \int_{0}^{t} \frac{f^{(m)}(\tau) d \tau}{(t-\tau)^{\alpha+1-m}}\right], \\
& =\frac{1}{\Gamma(m-\alpha)} \mathcal{L}\left[\int_{0}^{t} \frac{f^{(m)}(\tau) d \tau}{(t-\tau)^{\alpha+1-m}}\right], \\
& =\frac{1}{\Gamma(m-\alpha)} \mathcal{L}\left[\int_{0}^{t}(t-\tau)^{m-1-\alpha} f^{(m)}(\tau) d \tau\right], \\
& =\frac{1}{\Gamma(m-\alpha)} \mathcal{L}\left[t^{m-\alpha-1}\right] \mathcal{L}\left[f^{(m)}(t)\right], \quad(\text { dari Teorema } 2.3), \\
& =\frac{1}{\Gamma(m-\alpha)} \frac{\Gamma(m-\alpha)}{s^{m-\alpha}}\left[s^{m} F(s)-\sum_{k=1}^{m} s^{(m-k)} f^{(k-1)}(0)\right], \\
& =\frac{1}{s^{m-\alpha}}\left[s^{m} F(s)-\sum_{k=1}^{m} s^{(m-k)} f^{(k-1)}(0)\right], \\
& =s^{\alpha} F(s)-\sum_{k=1}^{m} s^{(\alpha-k)} f^{(k-1)}(0) .
\end{aligned}
$$

\section{Pembahasan}

Perhatikan kembali sistem persamaan (1.1), yaitu

$$
\frac{d^{\alpha} \mathbf{x}(t)}{d t^{\alpha}}=A \mathbf{x}(t)+B \mathbf{u}(t), \mathbf{x}(0)=\mathbf{x}_{0},
$$

dimana $A \in \mathbb{R}^{n \times n}, B \in \mathbb{R}^{n \times m}, \mathbf{x}(t) \in \mathbb{R}^{n}, \mathbf{u}(t) \in \mathbb{R}^{m}$ dan $\frac{d^{\alpha} \mathbf{x}(t)}{d t^{\alpha}}$ menyatakan turunan fractional orde $\alpha$ dari $\mathbf{x}(t)$.

Teorema 3.1. [3] Solusi dari sistem persamaan (1.1) untuk $m-1<\alpha<m$, $m \in \mathbb{N}$ adalah

$$
\boldsymbol{x}(t)=\sum_{k=1}^{m} \Phi_{k}(t) \boldsymbol{x}^{(k-1)}(0)+\int_{0}^{t} \Phi(t-\tau) B \boldsymbol{u}(\tau) d \tau,
$$

dimana

$$
\begin{aligned}
\Phi_{k}(t) & =\sum_{n=0}^{\infty} A^{n} \mathcal{L}^{-1}\left[s^{-(\alpha n+k)}\right]=\sum_{n=0}^{\infty} \frac{A^{n} t^{(n \alpha+k)-1}}{\Gamma(n \alpha+k)} \\
\Phi(t) & =\sum_{n=0}^{\infty} A^{n} \mathcal{L}^{-1}\left[s^{-(n+1) \alpha}\right] \\
& =\sum_{n=0}^{\infty} \frac{A^{n} t^{(n+1) \alpha-1}}{\Gamma(n+1) \alpha}
\end{aligned}
$$


104 Nadifa Rahadatul 'Aisy dkk

Bukti. Dengan menerapkan transformasi Laplace pada sistem (1.1), diperoleh

$$
\begin{aligned}
\mathcal{L}\left[\frac{d^{\alpha}}{d t^{\alpha}} \mathbf{x}(t)\right] & =\mathcal{L}[A \mathbf{x}(t)+B \mathbf{u}(t)] \\
& =\mathcal{L}[A \mathbf{x}(t)]+\mathcal{L}[B \mathbf{u}(t)]
\end{aligned}
$$

Berdasarkan persamaan (2.3) pada Teorema 2.8, diperoleh

$$
\begin{aligned}
s^{\alpha} \mathbf{X}(s)-\sum_{k=1}^{m} s^{(\alpha-k)} \mathbf{x}^{(k-1)}(0) & =A \mathbf{X}(s)+B \mathbf{U}(s), \\
s^{\alpha} \mathbf{X}(s)-A \mathbf{X}(s) & =B \mathbf{U}(s)+\sum_{k=1}^{m} s^{(\alpha-k)} \mathbf{x}^{(k-1)}(0), \\
\left(I_{n} s^{\alpha}-A\right) \mathbf{X}(s) & =B \mathbf{U}(s)+\sum_{k=1}^{m} s^{(\alpha-k)} \mathbf{x}^{(k-1)}(0) .
\end{aligned}
$$

Sehingga,

$$
\mathbf{X}(s)=\left[I_{n} s^{\alpha}-A\right]^{-1}\left[B \mathbf{U}(s)+\sum_{k=1}^{m} s^{(\alpha-k)} \mathbf{x}^{(k-1)}(0)\right]
$$

Karena

$$
\begin{aligned}
{\left[I_{n} s^{\alpha}-A\right]^{-1} } & =\left[\frac{I}{s^{\alpha}}+\frac{A}{s^{2 \alpha}}+\frac{A^{2}}{s^{3 \alpha}}+\cdots+\frac{A^{n}}{s^{(n+1) \alpha}}+\cdots\right] \\
& =\sum_{n=0}^{\infty} A^{n} s^{-(n+1) \alpha}
\end{aligned}
$$

maka persamaan (3.4) menjadi

$$
\begin{aligned}
\mathbf{X}(s) & =\sum_{n=0}^{\infty} A^{n} s^{-(n+1) \alpha}\left[B \mathbf{U}(s)+\sum_{k=1}^{m} s^{(\alpha-k)} \mathbf{x}^{(k-1)}(0)\right] \\
& =\sum_{n=0}^{\infty} A^{n} s^{-(n+1) \alpha} \sum_{k=1}^{m} s^{(\alpha-k)} \mathbf{x}^{(k-1)}(0)+\sum_{n=0}^{\infty} A^{n} s^{-(n+1) \alpha} B U(s), \\
& =\sum_{n=0}^{\infty} \sum_{k=1}^{m} A^{n} s^{-(\alpha n+k)} \mathbf{x}^{(k-1)}(0)+\sum_{n=0}^{\infty} A^{n} s^{-(n+1) \alpha} B U(s)
\end{aligned}
$$

Dengan mentransformasi Laplace, inverskan $\mathbf{X}(s)$ diperoleh

$$
\begin{aligned}
\mathbf{x}(t) & =\mathcal{L}^{-1}[\mathbf{X}(s)] \\
& =\mathcal{L}^{-1}\left[\sum_{n=0}^{\infty} \sum_{k=1}^{m} A^{n} s^{-(\alpha n+k)} \mathbf{x}^{(k-1)}(0)+\sum_{n=0}^{\infty} A^{n} s^{-(n+1) \alpha} B U(s)\right], \\
& =\mathcal{L}^{-1}\left[\sum_{n=0}^{\infty} \sum_{k=1}^{m} A^{n} s^{-(\alpha n+k)} \mathbf{x}^{(k-1)}(0)\right]+\mathcal{L}^{-1}\left[\sum_{n=0}^{\infty} A^{n} s^{-(n+1) \alpha} B U(s)\right],
\end{aligned}
$$




$$
\begin{aligned}
& =\sum_{n=0}^{\infty} \sum_{k=1}^{m} A^{n} \mathcal{L}^{-1}\left[s^{-(\alpha n+k)}\right] \mathbf{x}^{(k-1)}(0)+\sum_{n=0}^{\infty} A^{n} \mathcal{L}^{-1}\left[s^{-(n+1) \alpha} B U(s)\right], \\
& =\sum_{n=0}^{\infty} \sum_{k=1}^{m} \frac{A^{n} t^{(n \alpha+k)-1}}{\Gamma(n \alpha+k)} \mathbf{x}^{(k-1)}(0)+\sum_{n=0}^{\infty} A^{n} \mathcal{L}^{-1}\left[s^{-(n+1) \alpha} B \mathbf{U}(s)\right], \\
& =\sum_{n=0}^{\infty} \sum_{k=1}^{m} \frac{A^{n} t^{(n \alpha+k)-1}}{\Gamma(n \alpha+k)} \mathbf{x}^{(k-1)}(0)+\int_{0}^{t} \sum_{n=0}^{\infty} A^{n}(t-\tau)^{(n+1) \alpha-1} B \mathbf{u}(\tau) d \tau .
\end{aligned}
$$

Dengan demikian,

$$
\mathbf{x}(t)=\sum_{k=1}^{m} \Phi_{k}(t) \mathbf{x}^{(k-1)}(0)+\int_{0}^{t} \Phi(t-\tau) B \mathbf{u}(\tau) d \tau
$$

dimana

$$
\begin{aligned}
\Phi_{k}(t) & =\sum_{n=0}^{\infty} A^{n} \mathcal{L}^{-1}\left[s^{-(\alpha n+k)}\right]=\sum_{n=0}^{\infty} \frac{A^{n} t^{(n \alpha+k)-1}}{\Gamma(n \alpha+k)} \\
\Phi(t) & =\sum_{n=0}^{\infty} A^{n} \mathcal{L}^{-1}\left[s^{-(n+1) \alpha}\right] \\
& =\sum_{n=0}^{\infty} \frac{A^{n} t^{(n+1) \alpha-1}}{\Gamma(n+1) \alpha}
\end{aligned}
$$

Contoh 3.2. Tentukanlah solusi dari sistem (1.1) dengan

$$
A=\left[\begin{array}{lll}
0 & 1 & 0 \\
0 & 0 & 0 \\
1 & 0 & 0
\end{array}\right], B=\left[\begin{array}{c}
1 \\
-2 \\
2
\end{array}\right], \mathbf{x}(0)=\left[\begin{array}{l}
1 \\
1 \\
1
\end{array}\right], \mathbf{x}^{\prime}(0)=\left[\begin{array}{c}
-1 \\
1 \\
-1
\end{array}\right], \mathbf{x}^{\prime \prime}(0)=\left[\begin{array}{c}
1 \\
0 \\
-1
\end{array}\right]
$$

untuk $\alpha=3 / 2$, dan $u(t)=1$.

Karena

$$
A=\left[\begin{array}{lll}
0 & 1 & 0 \\
0 & 0 & 0 \\
1 & 0 & 0
\end{array}\right], \quad \text { maka } A^{n}=\left[\begin{array}{lll}
0 & 0 & 0 \\
0 & 0 & 0 \\
0 & 0 & 0
\end{array}\right], \quad \text { untuk } n=3,4,5, \cdots .
$$

Dengan menggunakan persamaan (3.2) dan (3.3), diperoleh

$$
\begin{aligned}
\Phi_{k}(t) & =\sum_{n=0}^{\infty} \frac{A^{n} t^{(n \alpha+k)-1}}{\Gamma(n \alpha+k)}, \\
& =\frac{A^{0} t^{(k)-1}}{\Gamma(k)}+\frac{A t^{(\alpha+k)-1}}{\Gamma(\alpha+k)}+\frac{A^{2} t^{(2 \alpha+k)-1}}{\Gamma(2 \alpha+k)}, \\
\Phi(t) & =\sum_{n=0}^{\infty} \frac{A^{n} t^{(n+1) \alpha-1}}{\Gamma(n+1) \alpha}, \\
& =I_{3} \frac{t^{\alpha-1}}{\Gamma(\alpha)}+A \frac{t^{2 \alpha-1}}{\Gamma(2 \alpha)}+A^{2} \frac{t^{3 \alpha-1}}{\Gamma(3 \alpha)} .
\end{aligned}
$$

Untuk $\alpha=\frac{3}{2}$, diperoleh $m=2$ karena $1<\frac{3}{2}<2$. 
Subtitusikan persamaan (3.6) dan (3.7) dengan $u(t)=1$ ke dalam persamaan (3.1), maka diperoleh

$$
\begin{aligned}
& \mathbf{x}(t)=\sum_{k=1}^{m} \Phi_{k}(t) \mathbf{x}^{(k-1)}(0)+\int_{0}^{t} \Phi(t-\tau) B \mathbf{u}(\tau) d \tau \\
& =\sum_{k=1}^{2} \Phi_{k}(t) \mathbf{x}^{(k-1)}(0)+\int_{0}^{t} \Phi(t-\tau) B \mathbf{u}(\tau) d \tau, \\
& =\Phi_{1}(t) \mathbf{x}(0)+\Phi_{2}(t) \mathbf{x}^{\prime}(0)+\int_{0}^{t} \Phi(t-\tau) B \mathbf{u}(\tau) d \tau, \\
& =\left[I_{3}+\frac{A t^{\alpha}}{\Gamma(\alpha+1)}+\frac{A^{2} t^{2 \alpha}}{\Gamma(2 \alpha+1)}\right] \mathbf{x}(0)+\left[\frac{A^{0} t^{1}}{\Gamma(2)}+\frac{A t^{\alpha+1}}{\Gamma(\alpha+2)}+\frac{A^{2} t^{2 \alpha+1}}{\Gamma(2 \alpha+2)}\right] \mathbf{x}^{\prime}(0), \\
& +\int_{0}^{t}\left[\frac{B}{\Gamma(\alpha)}(t-\tau)^{\alpha-1}+\frac{A B}{\Gamma(2 \alpha)}(t-\tau)^{2 \alpha-1}+\frac{A^{2} B}{\Gamma(3 \alpha)}(t-\tau)^{3 \alpha-1}\right] d \tau, \\
& =\mathbf{x}(0)+\frac{A \mathbf{x}(0) t^{\alpha}}{\Gamma(\alpha+1)}+\frac{A^{2} \mathbf{x}(0) t^{2 \alpha}}{\Gamma(2 \alpha+1)}+\frac{A^{0} \mathbf{x}^{\prime}(0) t^{1}}{\Gamma(2)}+\frac{A \mathbf{x}^{\prime}(0) t^{\alpha+1}}{\Gamma(\alpha+2)}+\frac{A^{2} \mathbf{x}^{\prime}(0) t^{2 \alpha+1}}{\Gamma(2 \alpha+2)}, \\
& +\frac{B t^{\alpha}}{\Gamma(\alpha+1)}+\frac{A B t^{2 \alpha}}{\Gamma(2 \alpha+1)}+\frac{A^{2} B t^{3 \alpha}}{\Gamma(3 \alpha+1)} \\
& =\left[\begin{array}{l}
1 \\
1 \\
1
\end{array}\right]+\left[\begin{array}{lll}
0 & 1 & 0 \\
0 & 0 & 0 \\
1 & 0 & 0
\end{array}\right]\left[\begin{array}{l}
1 \\
1 \\
1
\end{array}\right] \frac{t^{\alpha}}{\Gamma(\alpha+1)}+\left[\begin{array}{lll}
0 & 0 & 0 \\
0 & 0 & 0 \\
0 & 1 & 0
\end{array}\right]\left[\begin{array}{l}
1 \\
1 \\
1
\end{array}\right] \frac{t^{2 \alpha}}{\Gamma(2 \alpha+1)}+\left[\begin{array}{c}
-1 \\
1 \\
-1
\end{array}\right] \frac{t}{\Gamma(2)} \\
& +\left[\begin{array}{lll}
0 & 1 & 0 \\
0 & 0 & 0 \\
1 & 0 & 0
\end{array}\right]\left[\begin{array}{c}
-1 \\
1 \\
-1
\end{array}\right] \frac{t^{\alpha+1}}{\Gamma(\alpha+2)}+\left[\begin{array}{lll}
0 & 0 & 0 \\
0 & 0 & 0 \\
0 & 1 & 0
\end{array}\right]\left[\begin{array}{c}
-1 \\
1 \\
-1
\end{array}\right] \frac{t^{2 \alpha+1}}{\Gamma(2 \alpha+2)}+\left[\begin{array}{c}
1 \\
-2 \\
2
\end{array}\right] \frac{t^{\alpha}}{\Gamma(\alpha+1)} \\
& +\left[\begin{array}{lll}
0 & 1 & 0 \\
0 & 0 & 0 \\
1 & 0 & 0
\end{array}\right]\left[\begin{array}{c}
1 \\
-2 \\
2
\end{array}\right] \frac{t^{2 \alpha}}{\Gamma(2 \alpha+1)}+\left[\begin{array}{lll}
0 & 0 & 0 \\
0 & 0 & 0 \\
0 & 1 & 0
\end{array}\right]\left[\begin{array}{c}
1 \\
-2 \\
2
\end{array}\right] \frac{t^{3 \alpha}}{\Gamma(3 \alpha+1)}, \\
& =\left[\begin{array}{l}
1 \\
1 \\
1
\end{array}\right]+\left[\begin{array}{l}
1 \\
0 \\
1
\end{array}\right] \frac{t^{\alpha}}{\Gamma(\alpha+1)}+\left[\begin{array}{l}
0 \\
0 \\
1
\end{array}\right] \frac{t^{2 \alpha}}{\Gamma(2 \alpha+1)}+\left[\begin{array}{c}
-1 \\
1 \\
-1
\end{array}\right] \frac{t}{\Gamma(2)}+\left[\begin{array}{c}
1 \\
0 \\
-1
\end{array}\right] \frac{t^{\alpha+1}}{\Gamma(\alpha+2)}, \\
& +\left[\begin{array}{l}
0 \\
0 \\
1
\end{array}\right] \frac{t^{2 \alpha+1}}{\Gamma(2 \alpha+2)}+\left[\begin{array}{c}
1 \\
-2 \\
2
\end{array}\right] \frac{t^{\alpha}}{\Gamma(\alpha+1)}+\left[\begin{array}{c}
-2 \\
0 \\
1
\end{array}\right] \frac{t^{2 \alpha}}{\Gamma(2 \alpha+1)}+\left[\begin{array}{c}
0 \\
0 \\
-2
\end{array}\right] \frac{t^{3 \alpha}}{\Gamma(3 \alpha+1)} \\
& =\left[\begin{array}{c}
1-\frac{t}{\Gamma(2)}+\frac{2 t^{\alpha}}{\Gamma(\alpha+1)}+\frac{t^{\alpha+1}}{\Gamma(\alpha+2)}-\frac{2 t^{2 \alpha}}{\Gamma(2 \alpha+1)} \\
1+\frac{t}{\Gamma(2)}-\frac{2 t^{\alpha}}{\Gamma(\alpha+1)} \\
1-\frac{t}{\Gamma(2)}+\frac{3 t^{\alpha}}{\Gamma(\alpha+1)}-\frac{t^{\alpha+1}}{\Gamma(\alpha+2)}+\frac{2 t^{2 \alpha}}{\Gamma(2 \alpha+1)}+\frac{t^{2 \alpha+1}}{\Gamma(2 \alpha+2)}-\frac{2 t^{3 \alpha}}{\Gamma(3 \alpha+1)}
\end{array}\right],
\end{aligned}
$$




$$
=\left[\begin{array}{c}
1-t+\frac{8}{3} t \sqrt{\frac{t}{\Pi}}+\frac{4}{3} t^{2} \sqrt{\frac{t}{\Pi}}-\frac{1}{3} t^{3} \\
1+t-\frac{8}{3} t \sqrt{\frac{t}{\Pi}} \\
1-t+4 t \sqrt{\frac{t}{\Pi}}-\frac{15}{8} t^{2} \sqrt{\frac{t}{\Pi}}+\frac{1}{3} t^{3}+\frac{1}{24} t^{4}-\frac{64}{945} t^{4} \sqrt{\frac{t}{\Pi}}
\end{array}\right]
$$

\section{Kesimpulan}

Solusi sistem persamaan diferensial linier fractional pada persamaan (1.1) adalah:

$$
\begin{aligned}
\mathbf{x}(\mathbf{t}) & =\sum_{k=1}^{m} \Phi_{k}(t) \mathbf{x}^{(k-1)}(0)+\int_{0}^{t} \Phi(t-\tau) B \mathbf{u}(\tau) d \tau, \text { dimana } \\
\Phi_{k}(t) & =\sum_{n=0}^{\infty} A^{n} \mathcal{L}^{-1}\left[s^{-(\alpha n+k)}\right]=\sum_{n=0}^{\infty} \frac{A^{n} t^{(n \alpha+k)-1}}{\Gamma(n \alpha+k)}, \\
\Phi(t) & =\sum_{n=0}^{\infty} A^{n} \mathcal{L}^{-1}\left[s^{-(n+1) \alpha}\right]=\sum_{n=0}^{\infty} \frac{A^{n} t^{(n+1) \alpha-1}}{\Gamma(n+1) \alpha} .
\end{aligned}
$$

\section{Daftar Pustaka}

[1] Anton, H. 1991. Aljabar Linier Elementer. Edisi Kelima. Terjemahan Bahasa Indonesia. Erlangga, Jakarta.

[2] Fallahgoul. A, Sergio. Focardi, Frank. Fabozzi. 2017. Fractional Calculus and Fractional Processes with Applications to Financial Economics. Academic Press, Australia.

[3] Kaczorek, T. 2011. Selected Problem of Fractional Systems Theory. SpringerVerlag, Berlin Heidelberg.

[4] Milici. Constantin, Gheorghe. D, J. Tanreiro. 2019. Introduction to Fractional Differential Equations. Springer, Switzerland.

[5] Prayudi. 2007. Matematika Teknik Persamaan Diferensial, Transformasi Laplace, Deret Fourier. Graha Ilmu, Yogyakarta.

[6] Spiegel, M . R. 1999. Transformasi Laplace. Terjemahan Bahasa Indonesia. Erlangga, Jakarta. 